Cahiers de recherches médiévales

Le choix de la prose (XIIIe-XVe siècles)

\title{
Pourquoi Moÿse?
}

Comment les romans en prose essaient de racheter le Moÿse qu'on trouve dans le Joseph de Robert de Boron

\section{Elspeth Kennedy}

\section{OpenEdition}

1 Journals

Édition électronique

URL : https://journals.openedition.org/crm/1352

DOI : 10.4000/crm.1352

ISSN : 1955-2424

Éditeur

Honoré Champion

\section{Édition imprimée}

Date de publication : 30 octobre 1998

Pagination : 33-42

ISSN : 1272-9752

\section{Référence électronique}

Elspeth Kennedy, « Pourquoi Moÿse? », Cahiers de recherches médiévales [En ligne], 5 | 1998, mis en ligne le 01 octobre 2007, consulté le 15 décembre 2022. URL : http://journals.openedition.org/crm/ 1352 ; DOl : https://doi.org/10.4000/crm.1352

Ce document a été généré automatiquement le 15 décembre 2022.

Tous droits réservés 


\title{
Pourquoi Moÿse?
}

\author{
Comment les romans en prose essaient de racheter le Moÿse qu'on \\ trouve dans le Joseph de Robert de Boron
}

\section{Elspeth Kennedy}

1 Les réactions des romans en prose du XIII ${ }^{\mathrm{e}}$ siècle à l'égard d'un certain épisode dans le Joseph de Robert de Boron fournissent un exemple intéressant des façons dont ces romans tentent d'adapter des matériaux hérités des textes en vers. On a accordé une grande attention à la manière dont Robert de Boron a utilisé des parallèles bibliques dans le Joseph et dans le Merlin pour donner son sens plein à son thème des Trois Tables, c'est-à-dire la Table de la Cène, la Table du Graal et la Table Ronde. ${ }^{1}$ Néanmoins, le fait qu'il ait attribué le nom de Moÿse au personnage qui se trouve englouti quant le sol s'ouvre sous ses pieds au moment où il prend la place restée vide à la Table du Graal ne s'insère pas facilement dans le cadre habituel des parallèles bibliques utilisés dans le reste du Joseph pour structurer l'œuvre. Les méthodes par lesquelles les successeurs de Robert ont tenté de traiter cet épisode dans leur réécriture de l'histoire du Graal, intéressantes par la signification particulière donnée dans certaines versions au châtiment par le feu, suggèrent également qu'eux aussi ont trouvé déconcertant le rôle donné ici à Moÿse.

2 Chez Robert de Boron, dans l'épisode où Moÿse apparaît, Joseph d'Arimathie, voyageant avec le vase sacré que lui a remis le Christ, est parti avec ses compagnons vers un pays lointain. ${ }^{2}$ Pendant un certain temps tout se passe bien, mais lorsque les récoltes commencent à être mauvaises les compagnons de Joseph se plaignent de ne rien avoir à manger; ils demandent à Hebron de pousser Joseph à implorer l'aide de Dieu. Joseph s'agenouille devant le vase sacré et prie le ciel de le guider. Dieu lui dit que leurs problèmes viennent des péchés de certains membres de leur groupe. Il doit établir la Table du Graal en souvenir de la Table de la Cène. Le vase sacré, recouvert d'un linge (et dont il apprend plus tard que c'est le Graal), doit être placé sur la table, ainsi qu'un poisson. Joseph doit s'asseoir à la place de Jésus, mais une place, celle où Judas s'était assis, devra rester vide, jusqu'à l'arrivée du descendant de Bron destiné à l'occuper (2531-2536). Puis tous ceux qui croient en la Sainte Trinité et qui ont suivi les commandements de Dieu devront venir à la table, mais pas les autres. Joseph suit ces 
instructions. Ceux qui s'assoient à la table voient leurs désirs, physiques et spirituels, exaucés, tandis que les désirs de ceux qui ne le font pas restent inassouvis. Les compagnons de Moÿse, qui est au nombre de ceux qui n'ont pas pu prendre place à la table, demandent à Joseph de le laisser s'asseoir à la place vide. Moÿse est prié de s'approcher, mais averti du danger pour ceux qui ne sont pas dignes. Il va à la place vide, s'assied, et la terre s'ouvre et l'engloutit; c'est comme s'il n'avait jamais existé. Une voix explique sa disparition: Moÿse a trompé Joseph et a reçu sa punition. On n'entendra plus parler de lui jusqu'à l'arrivée de celui à qui la place vide était destinée :

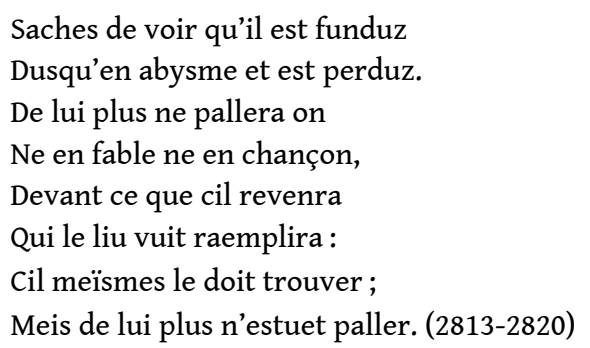

3 Cet épisode est clairement lié au thème des Trois Tables (ici la Table de la Cène et celle du Graal - Robert introduisant la Table Ronde dans son Merlin-), mais l'épisode gagne aussi une signification particulière par un parallèle avec l'Ancien Testament, la disparition semblable de Core, de Dathan et d'Abiron. Dans les Numeri 16, ces trois personnages se rebellent contre l'autorité de Moïse. Moïse déclare à Core et à sa troupe :

Mane, inquit, notum faciet Dominus qui ad se pertineant et sanctos applicabit sibi : et quos ellegerit, appropinquabunt ei. (Numeri 16,5)

Ils refusent de l'écouter et se plaignent de la façon dont il les dirige et des épreuves qu'ils ont traversées. Moïse leur dit alors que s'ils meurent d'une mort ordinaire, il n'est pas l'envoyé du Seigneur :

Sin autem novam rem fecerit Dominus, ut aperiens terra os suum deglutiat eos et omnia quae ad illos pertinent, descenderintque viventes in infernum, scietis quod blasphemaverint Dominum. Confestim igitur ut cessavit loqui, dirupta est terra sub pedibus eorum. Et aperiens os suum devoravit illos cum tabernaculis suis, et universa substantia eorum. Descenderuntque vivi in infernum operti humo, et perierunt de medio multitudinis. (vers 30-33)

5 Les encensoirs qu'ils avaient offerts sont remis à l'état de plaques d'argent qui recouvriront l'autel :

ut haberent postea filii Israeli quibus commonerentur, ne quis accedat alienigena, et qui non est de semine Aaron, ad offerendum incensum Domino, ne patiatur sicut passus est Core, et omnis congregatio ejus, loquente Domino ad Moysen. (vers 40)

6 Les parallèles sont clairs. On retrouve, bien sûr, le cadre général d'un groupe de gens qui ont la charge de quelque chose de saint dont les mystères ne sont pas exprimables en termes humains habituels (l'Arche d'Alliance/le Graal), qui sont conduits vers un pays étrange et nourris miraculeusement (la manne céleste, la nourriture sur la Table du Graal). Plus spécifique à l'épisode dont il est ici question, les doléances de ceux qui rencontrent des épreuves dans le désert. Il existe un élément commun important : c'est la séparation de ceux qui sont dignes de ceux qui ne le sont pas, et aussi la place réservée à ceux qui sont d'un certain lignage. et par-dessus tout, le terrible châtiment de quiconque veut absolument, malgré les avertissements, s'approcher de quelque chose de saint alors qu'il n'est pas suffisamment pur. Les noms utilisés sont intéressants. Le nom Joseph d'Arimathie maintient un lien avec le Nouveau Testament, 
bien qu'il annonce aussi des événements postérieurs au Nouveau Testament, et fournisse par ses descendants un lien avec le livre suivant dans l'œuvre de Robert de Boron, le Merlin. Le nom Hebron ou Bron a un lien très net avec l'Ancien Testament: Hebron descend d'Aaron et il est l'un de ceux qui ont la charge de l'Arche d'Alliance (Numeri 3). Mais pourquoi Moÿse? Moïse dans le Nouveau Testament, tout comme dans les interprétations médiévales de l'Ancien Testament serait non seulement le gardien de la Vieille Loi, mais aussi l'un de ceux qui ont préparé la voie pour la venue du Christ ; en vérité Élias et lui sont présents lors de la Transfiguration du Christ dans le Nouveau Testament. Tel est son rôle dans l'iconographie médiévale. Alors pourquoi donner ce nom, Moÿse, à quelqu'un qui reçoit un si terrible châtiment divin, alors que le système général de références dans Robert de Boron présente la Nouvelle Loi comme une transformation et un accomplissement des prophéties de la Vieille Loi plutôt qu'en termes d'une opposition entre les deux?

7 Dans les interprétations typologiques caractéristiques des textes médiévaux postérieurs - c'est-a-dire à peu près cent ans après Robert, comme dans par exemple la Biblia Pauperum -, la terre qui se fend pour engloutir Dathan et Abiron devient, comme la destruction de Sodome, une préfiguration des peines de l'Enfer. ${ }^{3}$ Les hérétiques étaient aussi comparés par les prédicateurs aux rebelles de l'Ancien Testament et menacés des mêmes châtiments. Hans Bayer, qui placerait l'ensemble du poème de Robert dans le contexte de la condamnation des hérésies comme celle des Cathares, voit dans Moÿse ici un hérétique dissimulé plutôt qu'un infidèle. ${ }^{4}$ Je ne suis pas entièrement convaincue qu'une attaque contre les hérésies contemporaines joue dans l'œuvre de Robert un rôle tout à fait aussi central que le soutient Bayer, mais de toute façon une telle théorie ne semble pas expliquer l'attribution du nom Moÿse à une personne condamnée de la sorte. Nitze (Joseph, p. XII) suggère que le nom peut être utilisé ici pour désigner l'incarnation de l'Ancienne alliance de Moïse par opposition avec la Nouvelle, une opposition normalement représentée par Synagoga et Ecclesia. Moÿse refuse d'accepter la Nouvelle alliance, en contraste avec Hebron qui en tant que gardien d'un objet abritant des mystères sacrés, remplit le même rôle par rapport au graal que son homonyme de l'Ancien Testament le fait par rapport à l'Arche d'Alliance. Mais cela semble être en contradiction avec l'emploi habituel des références bibliques à l'intérieur de l'œuvre. Dans le Merlin en prose, étroitement fondé sur le Merlin de Robert de Boron, si l'on en juge par la comparaison avec l'unique fragment qui nous soit parvenu sous la forme en vers originelle, lorsque Merlin informe Uter que la Table Ronde doit être établie, il explique au roi comment la Table du Graal a été établie en souvenir de la Table de la Cène et lui parle de la place vide, qui représente la place de Judas Iscariote. Cependant, dans le manuscrit édité par Micha, Merlin ne mentionne pas la disparition de Moÿse, bien que cet épisode trouve son écho dans l'engloutissement d'un homme dont l'identité n'est pas donnée, un baron puissant qui ose s'asseoir à la Table Ronde à la place réservée à celui qui est destiné à s'asseoir le premier à la place laissée vide à la Table du Graal. ${ }^{5}$ Comme Moÿse, le baron est un personnage qui trompe les autres pour pouvoir s'asseoir à cette place, mais Merlin refuse de dire ce qui lui est arrivé. Le sort de Moÿse, par conséquent, et la signification du nom qui lui a été donné, restent mystérieux dans les deux poèmes de Robert.

8 Venons-en maintenant à la façon dont d'autres romans en prose du début du XIII ${ }^{\mathrm{e}}$ siècle ont traité cet aspect particulier d'une œuvre à laquelle ils étaient confrontés au cours du processus de réécriture de l'histoire du Graal. La forme que prend cette interaction 
dans les romans du Graal plus tardifs signifie qu'une certaine familiarité avec les sources, la reconnaissance des allusions qu'on y fait, ainsi que de tous les changements qu'on y apporte, sont nécessaires à une pleine compréhension de la nouvelle création littéraire (tout à fait de la même façon que le Nouveau Testament ne peut pas être isolé de l'Ancien si l'on veut vraiment arriver à comprendre tout l'éventail de ses significations). C'est à leurs risques et périls que les médiévistes isolent tout roman du Graal de son intertexte.

9 Je voudrais tout d'abord examiner brièvement un autre texte en vers qui a eu une grande influence sur les romans en prose du Graal. Dans la Première Continuation $d u$ Perceval de Chrétien, on trouve un long passage, à la fois dans la version courte et dans la version longue, où on fait à Gauvain un récit largement basé sur Robert de Boron de l'histoire du Graal avant Merlin. ${ }^{6}$ Là, Joseph lui-même se rend tout droit à l'Isle Blanche (une partie de l'Angleterre), et lui et sa troupe y livrent bataille à leurs ennemis avant que Joseph ne remette le Graal au Riche Pescheor et à ses descendants; puis Joseph meurt. Il n'y a évidemment pas de Siège Périlleux, l'épreuve pour le héros du Graal consistant à poser des questions; en fait, les références bibliques sont pour le plus grand nombre au Nouveau Testament plutôt qu'a l'Ancien. Pour la première partie, on suit Robert de Boron tout à fait étroitement; mais après le départ de Terre Sainte, on ne rencontre plus les mêmes parallèles entre l'Arche d'Alliance et les errances du Graal, bien qu'il y ait un repas miraculeux associé au Graal (A 7615-7618); il n'est pas fait mention d'Hebron ou de Moÿse; Joseph reste présent, et entre dans la terre que Dieu lui a promise, l'Angleterre (A 7587-7588).

Dans le Perlesvaus, une autre sorte de continuation du Perceval (où il n'y a pas non plus de Siège Perilleux), de nouveau pas de Moÿse: on y met cependant emphatiquement l'accent sur l'opposition entre la Vieille Loi et la Nouvelle, mais à plus d'une occasion en termes de Sarrasins et de Chrétiens. ${ }^{7}$ Par exemple, la signification des têtes de chevaliers en or, en argent et en plomb, apportées dans une charrette à la cour d'Arthur par les trois demoiselles, est expliquée à Gauvain par un prêtre dans les termes suivants :

et li chief des chevaliers seelé en or senefient la Novele Loi, et li chief seelé en argent les Giués, et li chief seelé en plon la fause loi des Sarazins. De ces trois manieres de genz est establiz li mondes. (Perlesvaus, p. 109)

11 Ailleurs dans le Perlesvaus la Nouvelle Loi n'est pas présentée en termes de l'accomplissement de la Vieille Loi, mais en opposition à elle, un lien avec les Sarrasins étant souvent insinué. ${ }^{8}$

Le Didot Perceval ou Perceval en prose contient une quête du Graal par Perceval qui suit le Joseph et le Merlin de Robert, une quête qui développe les thèmes de Robert, celui des Trois Tables et celui du Siège Perilleux. ${ }^{9}$ J'ai attendu jusqu'à maintenant pour discuter de cette œuvre parce que le Perceval de Chrétien et la Deuxième Continuation en sont des sources importantes. Le héros imparfait, Perceval, est hérité de Chrétien et de la Deuxième Continuation, si bien que lorsque Perceval s'assied sur le Siège Perilleux à la Table Ronde, la pierre se fend sous lui avec un bruit terrible tandis qu'une voix rappelle à Arthur les instructions de Merlin, tout en disant que Perceval a fait quelque chose de bien risqué. Le roi s'entend dire :

Et bien saces que se ne fust por la bonté Alain le Gros son pere, et por la bonté Bron son taion, qui est clamés li Rois Peschiere, qu'il fust fondus en abisme et morust de 
la dolerouse mort dont Moÿs morut quant il s'asist fausement el liu que Joseph li avoit desfendu. (Didot Perceval, p. 150) ce pays et s'appelle le Graal. Il apprend que la faille dans le siège à la Table Ronde se ressoudera et que le Roi Pêcheur sera guéri quand le chevalier destiné à s'asseoir sur ce siège aura atteint le sommet de la chevalerie sur terre et aura posé les questions au sujet du Graal (Didot Perceval, pp. 150-151). Nulle mention n'est faite ici de Moÿse sauvé de l'abîme quand cela sera accompli, ce qui est impliqué par la phrase cil meïsmes (l'homme destiné à s'asseoir sur le siège à la Table du Graal) le doit trouver (Joseph, 2819). Jean Frappier a fait remarquer que certains manuscrits du Joseph en prose adaptent leur traduction de Robert pour qu'elle s'accorde avec le Perceval en prose encore à venir. ${ }^{10}$ Ainsi par rapport aux prédictions sur le sort de Moÿse, dans le Joseph en prose qu'on trouve dans Paris BN fr. 423, f. $44 \mathrm{~d}$, cette prophétie prend une forme plutôt vague : « Ne jamés de lui n'iert parolle tenue devant que cil qui le trovera le truit. Ne de lui ne sera plus parolle tenue.» Dans le Joseph du manuscrit de Modène la leçon prépare plus nettement le terrain au Perceval en prose dans lequel on ne mentionne absolument pas que Moÿse doive jamais être retrouvé : «Et bien saces que il est fondus en abisme ne de lui sera mais parole. $»^{11}$ Plus loin dans le récit, dans le Joseph (en vers) de Robert, la voix céleste annonce à Joseph que le tiers hom donnera des nouvelles du sort de Moÿse :

Dira li que est devenuz

Moysés qui estoit perduz (3135-3136)

The toute cette allusion à Petrus recevant des nouvelles est omise dans le Joseph du manuscrit Didot (Paris BN. n.a.fr. 4166), le Joseph du manuscrit de Modène et celui de B.N. fr. 423 gardent à cet endroit l'allusion, mais le nom Moÿse est remplacé par toy. :

Ne il ne porra aller de vie a mort devant qu'il ait celui qui le brief lira et enseignera

la force de ton vaissel, et cil qui vindra li dira novelles de toy. ${ }^{12}$

Ainsi dans le cycle en prose dérivé du Joseph et du Merlin de Robert avec un Perceval (et une Mort Artu basée sur les chroniques), le thème du Siège Perilleux qui engloutira le présomptueux dans l'abîme est développé plus avant, avec les modifications requises pour le héros moins-que-parfait hérité de Chrétien et de la Deuxième Continuation, mais Moÿse est encore laissé dans l'abîme, presque comme si le roman en prose n'était guère à l'aise avec un tel personnage portant ce nom.

Le cycle Lancelot-Graal sous sa forme la plus développée est étroitement lié à Robert de Boron par l'importance qu'il accorde au thème des Trois Tables; il est particulièrement intéressant par son système d'allusions à l'œuvre de Robert et par sa réécriture créative de cette source primordiale. Cependant le développement du cycle semble être passé par différentes étapes. A sa première étape, le récit de l'enfance de Lancelot et de ses aventures jusqu'au point où il se gagne un siège à la Table Ronde ne présente pas la quête du Graal comme faisant partie intégrante de l'histoire de Lancelot, parce que le héros ici, c'est encore Perceval, et pas Galaad. ${ }^{13}$ Les allusions qui font descendre le héros Lancelot de David, cité comme étant l'un des premiers grands chevaliers, s'insèrent bien dans le schéma familier qu'on trouve dans les prières utilisées lors de la cérémonie de la bénédiction des armes. Mais on trouve aussi des allusions qui en évoquant l'Ancien Testament font le lien avec Robert de Boron et qui nous rappellent l'interaction avec l'Ancien Testament qu'on trouve dans son Joseph. Ban, le père de Lancelot, à l'heure de sa mort, supplie Dieu d'avoir pitié de sa femme Hélène : 
Biax Peres piteus, preigne vos pitiez de ma fame Helene, qui est descendue del haut lignage que vos establites el Regne Aventureus a essaucier vostre non et la hautesce de vostre foi, et a avoir voz granz repostailles, qui devant les estranges pueples lor avez victoire donee (LK, pp. 13-14)

La référence aux granz repostailles qui ont donné aux ancêtres d'Hélène (la mère de Lancelot) la victoire sur les estranges pueples n'est jamais totalement expliquée, mais, prise en conjonction avec le fait qu'Hélène descend de David, elle amène des échos de l'Ancien Testament où l'Arche d'Alliance, qui contient le Témoignage de Dieu, les témoignages qui représentent les mystères de Dieu, a précédé dans la bataille les enfants d'Israël..$^{14}$ Ces allusions rappellent également les errances du Graal chez Robert de Boron, où le Graal représente l'Arche de la Nouvelle Alliance qui recèle les secrets de Dieu. Le lien avec Robert de Boron et à travers lui avec l'Ancien Testament est aussi clairement indiqué dans un passage décrivant une maison religieuse où Lancelot passe la nuit et dans laquelle se trouve la tombe de Leucain, un neveu de Joseph d'Arimathie :

En cele maison avoit une sepolture que l'an apeloit Leucain. Cil Leucanz fu niés Joseph de Darimathie, cel dont li granz lignages descendié par cui la Granz Bretaigne fu puis enluminee, car il i porterent lo Graal et conquistrent la terre mescreant a Nostre Seignor. (LK, p. 179)

Il faut comparer cela avec un passage dans le Joseph, où Joseph d'Arimathie remet le Graal au Riche Pescheor (Hebron/Bron) et lui dit qu'ils doivent l'emporter à l'ouest, mais sans qu'il soit question de batailles :

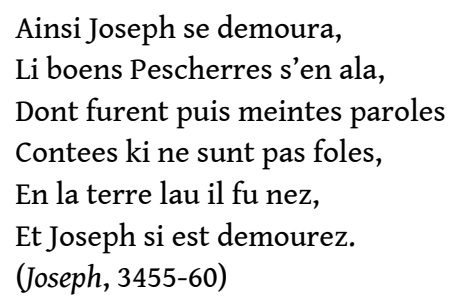

Dans l'explication donnée à Arthur par un preudomme concernant la flor qui parle, un des éléments de l'interprétation fournie par les sages clercs du roi d'un rêve qu'il a eu, le preudomme parle du fruit qui vient de la fleur :

C'est li fruiz don li cors est sostenuz et l'ame paüe. C'est li fruiz qui saola les cinc mile homes en la praerie qant les doze corboilles furent anplies del reillié. Ce est li fruiz par coi li pueples Israel fu sostenuz quinze anz es desserz, la ou li om, ce dit l'Escripture, manja lo pain as angles. Ce est li fruiz par coi Josep de Barimathia et si compaignon furent sostenu qant il s'an venoient de la terre de promission an cest estrange païs par lo comendement Jhesu Crist et par son conduit. Ce est li fruiz don Sainte Église est repaüe chascun jor. Ce est Jhesus Criz, li Filz Deu. (LK, p. 292)

Ce passage ne contient aucune référence à ce qui va se passer plus tard, mais crée un lien avec le miracle de la multiplication des pains qu'on trouve dans le Nouveau Testament, avec l'Exode où les enfants d'Israël reçoivent dans le désert la manne céleste (panes de coelo) Exodus $16: 4$ ) et avec le Joseph, où les élus reçoivent de la nourriture à la Table du Graal ; le passage souligne l'héritage ancestral de Lancelot par ces échos bibliques et insère son histoire dans le contexte de la tradition arthurienne déjà existante.

21 Une fois la décision prise de faire entrer ce premier Lancelot en prose dans un cycle qui comprend une Quête du Saint Graal dans laquelle un Siège Périlleux à la Table Ronde joue un rôle important, les aventures de Lancelot commencent à incorporer des épisodes qui ouvrent la voie à une Quête du Graal qui doit être une branche postérieure 
dans le même cycle. C'est dans un épisode de cette sorte que nous rencontrons la première allusion au personnage nommé Moÿse, mais plutôt que lui, c'est son neveu Symeu, un personnage que l'on ne rencontre pas dans Robert de Boron, qui a le droit de parler du fond de sa tombe et qui joue un rôle significatif par rapport au nouveau vainqueur de la Quête du Graal, Galaad. L'incident prend place dans la réécriture en prose du Chevalier de la Charrette de Chrétien. Lancelot trouve par hasard la tombe de son ancêtre Galaad (où il mène à bien l'aventure associée au tombeau) et celle de Symeu, où il rencontre l'échec. Symeu est donné ici comme étant le père de Moÿse, «a celui dont li cors gisoit en la Dolorose Tombe de la sale as grans poors, dont li grans contes parole del Graal. $»^{15}$ Des flammes sortent de la tombe de Symeu et il s'adresse à Lancelot qui ne peut pas s'approcher de la tombe; dans la Queste del saint Graal, Galaad éteint les flammes, Symeu s'adresse à lui, et Galaad porte son corps dans l'église où il est de nouveau enterré, mais à ce point de la Queste, il n'est pas fait mention de Moÿse. ${ }^{16}$ Et même, il y a dans la Queste (p.69), par contraste, une allusion favorable à ce chef de l'Ancien Testament, Moïse, qui fait jaillir l'eau du rocher. L'attribution du nom Moÿse à un personnage puni pour avoir eu l'audace de prétendre être digne de s'asseoir à la Table du Graal ne semble pas s'insérer confortablement dans le système d'allusions bibliques dans la Queste, où de grandes figures comme Salomon ont des rôles qui n'entrent pas en conflit avec l'interprétation typologique habituelle de l'Ancien Testament. L'opposition entre la Vieille Loi et la Nouvelle est illustrée par le rêve de Perceval dans lequel il voit deux femmes: une qui chevauche un serpent et l'autre un lion; ce rêve est interprété par un preudomme de la façon la plus traditionnelle : la femme qui chevauche un serpent c'est Synagoga, qui tient fermement à la Vieille Loi, et la femme qui chevauche un lion (Jésus Christ) représente la Nouvelle Loi, Ecclesia. Cependant on rencontre aussi à l'occasion, dans le Lancelot-Graal, l'opposition entre la Nouvelle Loi et les Sarrasins, opposition qui revêt une certaine importance dans le Perlesvaus. On nous dit par exemple que la foi de Mahommet prévalait en GrandeBretagne avant la venue de Joseph d'Arimathie. Quand Joseph part pour l'Écosse, le pays retombe sous le coup de la Loi Islamique, mais le roi qui est cause de tout cela et qui a envoyé au martyre les compagnons que Joseph avait laissés en Grande-Bretagne, tombe fou et se jette dans le feu. ${ }^{17}$

C'est seulement quand l'Estoire del Saint Graal est plus tard ajoutée pour fournir une branche qui ouvre le cycle Lancelot-Graal qu'on tente quelque peu dans cette réecriture massive du Joseph de Robert de donner à Moÿse un rôle qui ait un sens à l'intérieur de la structure de l'ensemble de l'œuvre. L'Estoire dans le cycle Vulgate réécrit et transforme l'épisode de Moÿse de Robert de Boron. On y raconte comment Joseph explique pourquoi le siège vide à la Table du Graal (qui symbolise la place de Jésus à la Table de la Cène) doit rester vide; c'est parce que ce siège attend son maître, Jésus Christ, ou quelqu'un qu'il enverra. Certains des personnages présents suggèrent que cela est faux, Symeu et Moÿse protestant encore plus fort que les autres. Moÿse obtient la permission d'essayer le siège ; il s'asseoit, simulant humilité et piété. Aussitôt sept mains brûlantes et enflammées le saisissent et le transportent dans les airs jusqu'à une forêt proche. ${ }^{18}$ Plus tard Josephus montre à ses compagnons un grand feu d'où sort la voix de Moÿse qui demande à Josephus de prier pour que ses souffrances soient plus légères et plus faciles à supporter. Moÿse explique comment, pendant qu'on l'emmenait, un preudome a obligé les mains à le reposer sur terre en disant :

Lessiez lo, car vos n'i avez droit, car il n'est mie vostres ; ne s'i n'a pas tant mesfait qu'il soit del tot perduz, einz trovera encore merci et pardon. Mais le meffet et 
l'outrage que il fist espeneïra il en tel maniere qu'il en sera en feu ardant en senefiance de ce qu'il a esté luxurieus jusq'a atant que li buens chevaliers, cil qui menra a fin les aventures de la Grant Bretaigne, le vendra visiter... (Estoire, tome II, p. 510)

Moÿse déclare, après que Josephus et Alain ont prié pour qu'il en soit ainsi, que ses souffrances sont adoucies, mais reconnaït cependant qu'il doit être puni. Le sort de Moÿse est donc ici modifié. Le sol ne s'ouvre pas sous lui pour l'engloutir comme c'est le cas pour Dathan et Abiron, dont le sort a été interprété comme étant une préfiguration de la damnation, du supplice des tourments de l'enfer. Moÿse est sauvé de la damnation éternelle, arraché au diable et brûlé par le feu comme s'il était au purgatoire, où ses souffrances sont adoucies par l'intercession des prières de Josephus et d'Alain.

Ainsi l'auteur de l'Estoire dans le cycle Lancelot-Graal essaie de régler le problème du manque d'explication concernant Moÿse dans la Queste et le transporte dans un lieu où il brûle comme en quelque Purgatoire, faisant disparaître sinon tous les éléments, du moins certains qui le lient à Dathan et Abiron, et à leur sort plus terrible encore que le sien. ${ }^{19}$ Comme Fanni Bogdanow l'a fait remarquer, dans certaines des versions postérieures de la Queste (B.N. f. fr. 343, la Demanda et le Tristan en prose), Moÿse n'est pas abandonné à ses souffrances dans sa tombe en flammes, mais, comme Symeu, il est délivré par Galaad. ${ }^{20}$ Mais même dans les versions qui introduisent une forme ou une autre de secours ou de soulagement pour Moÿse, le nom ne trouve toujours pas une place confortable dans le système de références à l'Ancien Testament, références qui préfigurent ce qui va se passer dans le Nouveau Testament, et système caractéristique de Robert de Boron et de la Queste. Je pense qu'il se peut fort bien que ce soit cette rupture avec le schéma traditionnel, conjuguée avec le fait que les romans en prose du $\mathrm{XIII}^{\mathrm{e}}$ siècle tendent habituellement à expliquer et à créer des liens nouveaux à l'intérieur d'un cycle, qui a mené à repousser Moÿse à l'arriere-plan en faveur d'un nouveau personnage nommé Symeu dans l'épisode de la Charrette et dans la Queste; ces épisodes rappellent les tourments de l'enfer et n'évoquent pas l'engloutissement de Dathan et d'Abiron dans l'Ancien Testament. Le récit dans l'Estoire tente, peut-être sans y réussir totalement, d'installer Moÿse dans cette réinterprétation, une réinterprétation qui semble, plutôt que faire la lumière sur l'utilisation de ce nom Moÿse par Robert de Boron, trahir une certaine gêne devant cette rupture avec le système habituel d'allusions à l'Ancien Testament qu'on trouve dans les romans $d u$ Graal au XIII siècle, et refléter peut-être aussi un intérêt croissant dans la doctrine du feu du Purgatoire.

\section{NOTES}

1. La façon dont la création littéraire de Robert a des interactions assez lointaines, à travers les traditions orales ou les livres auxquels il fait référence, avec la Bible à laquelle cette création est liée par des semblances typologiques, a été, au cours de ces dernières années, explorée avec succès par un certain nombre de chercheurs dont par 
exemple Emmanuèle Baumgartner, dans « Robert de Boron et l'imaginaire du livre du Graal », réimprimé dans De l'histoire de Troie au livre du Graal, Orléans, 1994, pp. 487-496.

2. Robert de Boron, Estoire dou saint Graal, éd. William A. Nitze, Paris, Champion, 1927 : abrégé en Joseph, vers 2361-2842.

3. Voir Louis Réau, Iconographie de l'art chrétien, tome II : Iconographie de la Bible I Ancien Testament, Paris, Presses Universitaires de France, 1956, p. 214.

4. Hans Bayer, Gral : Die hochmittelalterliche Glaubenskrise im Spiegel der Literatur, Stuttgart, 1983, p. 111.

5. Robert de Boron, Merlin : Roman du XIIIe siècle, éd. Alexandre Micha, Paris-Genève, Droz, 1979, pp. 193-196.

6. The Continuations of the Old French Perceval of Chrétien de Troyes, vol. III, Part I, The First Continuation, Redaction of MSS A L P R S, ed. William Roach, Philadelphia, 1952, A 7586-7604.

7. Le Haut Livre du Graal : Perlesvaus, ed. William A. Nitze and T. Atkinson Jenkins, Chicago, 1932-37.

8. Voir par exemple Perlesvaus, pp. 234, 235, 327.

9. The Didot Perceval according to the Manuscripts of Modena and Paris, ed. William Roach, Philadelphia, University of Pennsylvania Press, 1941.

10. Grundriss der romanischen Literaturen des Mittelalters, IV/1, Heidelberg, 1978, p. 533.

11. William Roach, «The Modena text of the Prose Joseph d'Arimathi », Romance Philology, IX

(1955-1956), pp. 313-342 (p. 337).

12. Romance Philology, IX, p. 340.

13. Voir Lancelot do Lac : the Non-Cyclic Old French Prose Romance, ed. Elspeth Kennedy, Oxford, Clarendon Press, 1980, vol. I, p. 33, Vol. II, pp. 89-90, abrégé en $L K$; Elspeth Kennedy, Lancelot and the Grail : a Study of the Prose Lancelot, Oxford, Clarendon Press, 1986, p. 150 ; Elspeth Kennedy, «Le Lancelot en prose (MS 45) », Les manuscrits français de la bibliothèque Parker: Parker Library, Corpus Christi College, Cambridge: Actes du Colloque 24-27 mars 1993, ed. Nigel Wilkins, Cambridge, Parker Library Publications, 1993, pp. 23-38.

14. Voir «Cumque elevaretur arca, dicebat Moyses : Surge, Domine, et dissipentur inimici tui, et fugiant qui oderunt te a facie tua. » (Num. 10 :35). voir aussi 2 Reg. 6 et 1 Par. 13-16.

15. Lancelot : roman en prose du XIIIe siècle, éd. Alexandre Micha, Genève-Paris, Droz, 1978-1983 (abrégé en LM), tome II, pp. 31-32).

16. La Queste del Saint Graal : roman en prose du XIIIe siècle, éd. Albert Pauphilet, Paris, Champion, 1923, pp. 264-265.

17. LM. tome II, pp. 253, 320-324.

18. L'Estoire del Saint Graal, ed. Jean-Paul Ponceau, Paris, Champion, 1997 (abrégé en Estoire), tome II, pp. 484-488.

19. Pour le développement des idées au sujet du Purgatoire aux XII ${ }^{\mathrm{e}}$ et XIII ${ }^{\mathrm{e}}$ siècles, voir Jacques le Goff, La naissance du Purgatoire, Paris, Éditions Gallimard, 1981.

20. Fanni Bogdanow, The Romance of the Grail, Manchester, Manchester University Press, 1966, pp. 101-104 ; Le roman de Tristan en prose, tome IX, ed. Laurence Harf-Lancner, Genève, Droz, 1997, pp. 209-210. 